\title{
Capítulo X \\ Innovación social, cambio tecnológico y gobierno abierto: la coproducción de políticas públicas
}

Joan Subirats

\section{Introducción}

Todo gran cambio tecnológico, como el que representan Internet y la revolución digital, ha acabado teniendo consecuencias en las formas de relación social y la configuración y modos de operar de las instituciones que acaban consolidándose. En la época contemporánea, esto sucedió tras el gran cambio que supuso la máquina de vapor y el impacto que implicó la revolución fordista-taylorista de inicios del siglo XX. No será Internet una excepción. Al contrario, sus efectos ya se dejan sentir mucho más que en los cambios mencionados, en la esfera de los servicios a personas y la administración de procesos y documentos. Obviamente, ello afecta a la política y a las políticas, a las instituciones democráticas, a los gobiernos y a las administraciones públicas. Por tanto, la literatura y los conceptos que se han utilizado deberán cambiar y acomodarse a nuevos actores, dinámicas, conflictos y roles.

Cabe recordar que, si se toma el momento fundacional de la perspectiva propia del análisis de políticas públicas (Laswell, Lindblom, Wildavsky, entre otros), y se lo relaciona con lo que era la agenda de los 
poderes públicos, se entenderá que sea posible hablar de una notable relación entre la expansión de las capacidades de intervención de las instituciones democráticas y la necesidad de contar con mejores y específicos instrumentos analíticos para seguir, comprender y evaluar la efectividad de tal intervención (Parsons, 1995; Peters, 2012). Lo que en los Estados Unidos se relacionó con los efectos del "Nuevo Pacto" (New Deal) o con los grandes programas de las Administraciones Kennedy y Johnson, en Europa se vinculó con la expansión de las políticas asociadas al Estado de bienestar y la estrechez que generaba una perspectiva administrativa muy marcada por la lógica jurídica y burocrática (Mayntz y Scharpf, 1975). Por su parte, en América Latina, se relacionó con la creciente atención de las necesidades sociales en un continente con grandes desigualdades y tensiones políticas (Oszlak y otros, 1976).

Se podría decir que hay una conexión notable entre producción de masas (democratización del consumo), democratización de los regímenes políticos (entendida como una mayor atención a las demandas sociales de servicios básicos, una mayor asunción de responsabilidades por parte de los poderes públicos en relación con tales necesidades y más apertura de los sistemas de toma de decisiones) y la necesidad de contar con instrumentos analíticos que tengan en cuenta esos cambios. Las bases analíticas se construyeron desde lógicas racionalizadoras y con fuertes sesgos normativos o de mejora de la eficacia y eficiencia en la acción pública. En general, se ha venido abordando una política pública desde lo que sería su lógica de acción, situándola en un escenario relativamente estable en que interactúan los actores político-administrativos y sociales. Al mismo tiempo, siempre se ha querido tener en cuenta las variables institucionales y la gama de distintos recursos que usan los actores para defender sus intereses (Subirats, 1989; Subirats y otros, 2008). El marco en que se situaba tal perspectiva analítica era y es esencialmente el de una democracia representativa, en que las elecciones sirven de base legitimadora de la acción institucional en un Estado-nación específico, y las capacidades de decisión de los poderes públicos resultan clave para configurar la política pública en cada caso.

En los últimos tiempos, los procesos de europeización de las políticas o la creciente influencia del escenario global en la formulación de políticas han ido generando matices y cambios de perspectiva (Radaelli, 2003; Schmidt y Radaelli, 2004; Reinicke y Copeland, 1998). Se han incorporado miradas y análisis que tienen en cuenta el impacto de la dimensión intergubernamental en la conformación de las políticas. Sin embargo, en general, se han mantenido las claves esenciales del esquema de análisis tradicional de las políticas públicas. 
En esta contribución, habría que tratar de situar los retos que plantea el cambio tecnológico de Internet y su creciente influencia en todo tipo de esferas de acción e interacción, tanto económica como, sobre todo, social y política. No se puede hablar solo de "crisis" para referirse a lo que sucede, ya que ello implicaría partir de una perspectiva de corta temporalidad, episódica, circunstancial. Como si solo se necesitara modular las respuestas ante una situación coyuntural de crisis, cuando en realidad aquí se parte de la convicción de que, después de Internet, las cosas ya no serán lo mismo. No basta con modular las respuestas, hay que replantearse las preguntas y los protagonismos. Por lo tanto, lo que se quiere es ver hasta qué punto tales transformaciones, que son al mismo tiempo motores de cambio muy relevantes en los escenarios políticos de todo el mundo, no exigen un replanteamiento de la forma de pensar, diseñar e implementar las políticas públicas. Todo ello sin perder de vista, al mismo tiempo, cómo esto influye en la distribución de poder y en los mecanismos (institucionales o no) de toma de decisiones públicas (Dente y Subirats, 2014).

\section{A. ¿Cambio de época o crisis de la intermediación política?}

Uno de los argumentos clave para defender la necesidad de replantear la perspectiva analítica sobre la que se ha construido el estudio de las políticas públicas es considerar, como se acaba de argumentar, que las sacudidas actuales que afectan al funcionamiento de las democracias no son algo meramente coyuntural. Lo que estaría aconteciendo es un proceso de transición o interregno entre dos sistemas distintos de producir, comunicarse, relacionarse y vivir (Bauman, 2012; Subirats, 2011). Las modificaciones que va incorporando Internet en nuestras vidas, tienen, como se ha mencionado, la dimensión que tuvieron revoluciones industriales y productivas como las de la incorporación de la máquina de vapor o, más tarde, el asentamiento del modelo taylorista-fordista de producción de masas (The Economist, 2012). Cada una de esas grandes transformaciones productivas vinieron acompañadas de cambios muy notables en las formas de convivencia y de relación social (por ejemplo, la formación de grandes aglomeraciones urbanas, estructuras familiares nucleares, división sexual del trabajo, estructura de clases vinculada a las estructuras industriales) y también cambios muy significativos en las instituciones políticas y las relaciones entre el poder político y la sociedad (estado liberal, democratización de estructuras representativas, partidos y sindicatos de masa). 
Internet representa una ruptura que, según el sector de que se trate, puede ser más o menos traumática con esta evolución productiva y económica (Benkler, 2015). En efecto, la nueva conectividad ha facilitado enormemente el tránsito hacia la financiarización global de la economía y ha permitido grandes procesos de deslocalización industrial. Sienta las bases para otros formatos de producción y de generación de valor que no tienen que estar vinculados forzosamente a la propiedad. Internet está provocando la amortización de un importante número de puestos de trabajo y modifica las relaciones laborales al permitir procesos generalizados de precarización y de autoempleo. Podría decirse que erosiona y pone en cuestión todos aquellos espacios de intermediación que no aportan valor propio y pueden quedar desplazados por la conexión directa entre proveedores y demandantes, o por el surgimiento de mecanismos de intermediación nuevos y más eficientes. Así pues, produce efectos de desintermediación y también de reintermediación (Chircu y Kauffman, 1999). Sin duda, ello afecta también a los espacios institucionales (de intermediación política) de la democracia representativa (Hacker y Van Dijk, 2000; Subirats, 2002; Edwards, 2006).

En ese sentido, conviene situar los aspectos más críticos de esa desintermediación. Los medios de comunicación tradicionales son los actores que más directa y visiblemente sufren este proceso, en la medida en que pierden control sobre las dinámicas de generación y difusión de información (fenómenos de productores-consumidores de información, a través de las redes sociales) y no logran incorporar entre sus seguidores a las nuevas generaciones digitalizadas (Gellman, 1996; Castells, 2009). No obstante, el aspecto central de esa amenaza de desintermediación radica en los actores que ocupan posiciones centrales de vínculo o conexión directa entre la sociedad, la ciudadanía y las instituciones políticas. Esto se refiere obviamente a los partidos políticos y a los grupos de interés como eslabones esenciales en esa conexión. Mediante los procesos electorales, esos factores acaban legitimando toda la arquitectura del poder político representativo. A través de los partidos, de las campañas electorales y del trabajo que se realiza entre elecciones, los poderes públicos han conocido tradicionalmente las necesidades que tenían los ciudadanos, así como sus preferencias en cuanto a la resolución de dichas necesidades. Los grupos de interés son los que han ido canalizando y organizando las distintas preferencias que se generaban en cada área de actividad, en busca de capacidad de influencia en las decisiones públicas que les afectaban (Edwards, 2006). Hoy en día, tanto la capacidad de producción de señales sobre lo que sucede, como su distribución y multiplicación, están en manos de los propios ciudadanos, sin que para ello tengan que pasar por determinados canales. Por otro lado, la gran cantidad de información disponible puede ser analizada y trabajada para conocer lo que acontece 
sin que sea necesario acudir a quienes tenían encomendada esa misión o posición. Existe la capacidad de recuperar el control, pero también es evidente que se han erosionado notablemente los centros de control que antes eran mucho más estables y fiables.

Diríase que la información, la comunicación y la representación son los espacios clave de la política de las instituciones democráticas en los que se están notando más los efectos del cambio tecnológico que conlleva Internet y la crisis de intermediación que genera. Es obvio que ha habido y hay intentos de recuperar posiciones en la nueva realidad de Internet, pero también es cierto que la perspectiva más común no acaba de integrar el cambio que ello representa en las propias estructuras organizativas e institucionales. Simplemente se pretende seguir haciendo la misma política de siempre, pero con un nuevo instrumento denominado Internet. Como afirma Mark Poster, Internet no es un martillo nuevo para seguir clavando los mismos clavos de siempre, de manera más cómoda y sencilla. Internet es una nueva realidad, una nueva configuración de realidades sociales, económicas y políticas (Poster, 2007). No parece posible, al menos a mediano plazo, tratar de encarar lo que implica mediante un simple proceso de acomodación instrumental.

En efecto, las características esenciales de lo que se podría denominar un sistema de gobierno "tradicional" (propio del modelo industrial-fordista y de un sistema político de democracia representativa) se podrían resumir en Estado-nación, gobierno representativo, principio de jerarquía, especialización competencial y administración burocrática.

La delimitación territorial del Estado define su ámbito de soberanía y el conjunto de la población a la que afecta tal ejercicio de autoridad soberana (Jellinek, 1978). Ese alcance territorial es también la esfera en que se identifican problemas y se buscan alternativas de solución. Además, se trata de formas de gobierno basadas únicamente en un modelo de democracia liberal-representativa, propio de la modernidad. Los gobernantes son elegidos, mediante un sistema de representación, para tomar decisiones y asumir la responsabilidad de las decisiones públicas (Pitkin, 2014). Las elecciones periódicas y competitivas son el principal (y prácticamente único) mecanismo mediante el cual el pueblo soberano podría controlar la acción de los gobernantes. También es clave el principio de jerarquía. El Estado, que es el único decisor público, toma y ejecuta sus decisiones con una lógica jerárquica, que le sirve tanto en su organización interna desde el punto de vista de su relación con otras esferas de gobierno de alcance territorial menor, como en sus relaciones con los ciudadanos y los actores que pueden representar sus intereses y necesidades. La organización del Estado y del ejercicio de su poder se basa en el 
principio de especialización competencial, que tiende a segmentar de tal manera los problemas y los destinatarios de las decisiones públicas, que difícilmente se recoge la complejidad e interrelación de tales asuntos. La forma de proceder de las distintas instancias gubernamentales es básicamente burocrática. Se despersonalizan los servicios y se busca una homogeneidad normativa y de proceso reglado que pocas veces se adapta a una realidad cada vez más heterogénea y diversificada.

En esa estructura, descrita esquemáticamente, son muy importantes y sustanciales los impactos de la transformación tecnológica que conlleva Internet y las dinámicas de globalización íntimamente vinculadas a su propia consolidación (Di Maggio y otros, 2001; Chadwick y Howard, 2009), aunque existan aún notables interrogantes sobre su real significación y su capacidad de alterar los equilibrios de poder hacia formatos de mayor profundización democrática (Morozov, 2012; 2014). Parece claro que las lógicas estrictas de Estado-nación son difíciles de mantener en un espacio cada vez más interrelacionado y en el que resulta imposible mantener aislamientos poco consistentes con el espacio digital. Sin duda, ello plantea temas relevantes desde el punto de vista del ejercicio de la soberanía en un ámbito territorial que no coincide con el espacio comunicativo y de interacciones sociales. Como ya se ha indicado, el principio representativo resulta especialmente afectado por una tecnología que no respeta los canales establecidos para relacionar realidad e instituciones políticas. Así, la potencialidad de desintermediación que permite Internet resulta más contradictoria con lógicas y procesos que preveían etapas y canales que ahora se transgreden fácilmente. El propio principio de la imposibilidad de decisión directa por parte del cuerpo social y la necesidad de articular estructuras representativas para hacerlo posible es un tema resoluble desde el punto de vista técnico. Ello genera constantes problemas con un ciclo electoral que establece plazos de mandato relativamente largos y estables y dinámicas propias del escenario actual, mucho más rápidas, que aceleran procesos y desestabilizan mandatos. Ese conjunto de procesos disruptivos en relación con las pautas establecidas en los parámetros de la democracia representativa se manifiestan además en los procesos de funcionamiento competencial y burocrático que tienen crecientes dificultades para mantener en pie sus previsiones procedimentales.

En definitiva, se puede discutir el grado en que esa dinámica de cambio tecnológico y social podrá modificar finalmente, de manera sustantiva y decisiva, la forma de funcionar de la democracia representativa. De lo que no cabe duda es de que las tensiones y los conflictos entre el "dentro" y el "fuera" de las instituciones aumentan con cada día que pasa. 


\section{B. Políticas públicas e Internet}

¿Cómo queda afectado el proceso de formación e implementación de las políticas públicas? Si bien el autor ya ha tratado este tema con anterioridad en términos generales (Subirats, 2012), convendría aquí abordar aquellos aspectos que marcan un antes y un después en el tema, y que hacen difícil sostener la hipótesis de una simple acomodación del esquema tradicional generalmente utilizado.

Como es sabido, el ciclo de las políticas públicas en sus distintas variantes (agenda, formulación, decisión o adopción de la política, implementación y evaluación), sigue usándose tanto descriptiva como analíticamente. Las críticas vertidas contra su linealidad, y su excesiva simplificación de lo que son procesos mucho más complejos y desordenados, no han logrado revertir su uso. No obstante, al margen de la utilidad del esquema y de su mayor o menor adecuación a la realidad, lo que parece claro es que en cualquier aproximación a la configuración de las políticas públicas se mezclan de manera más ordenada o menos lineal, problemas, alternativas, agenda, decisiones, puesta en práctica y evaluación. Se distinguen diferentes posiciones, intereses y estrategias de actores, sean de estatuto público o no. Se sabe que esa visión aparentemente pluralista esconde una asimetría de recursos, diversidad de roles y mayor o menor capacidad de incidencia en todo el proceso.

Lo que resulta más claramente afectado por el nuevo espacio digital propiciado por Internet es la arquitectura general de posiciones, estatus, distribución de recursos y niveles, que caracterizaban de manera muy estable las diversas comunidades normativas configuradas en torno a cada política, a cada especialización competencial y a cada una de las estructuras políticoadministrativas. Internet ha removido y modificado esa arquitectura, sin que aún sea posible referirse a un nuevo artefacto de formación de políticas. Además, ha quedado alterada la posición de centralidad institucional que mantenía la capacidad formal de tomar decisiones que concluían debates y abrían dinámicas de implementación. Como ya se ha mencionado, los fenómenos de desintermediación y reintermediación se suceden continuamente en los escenarios sociales, sean mercantiles o no. Surgen constantemente nuevas iniciativas que plantean formas de resolver problemas o encarar situaciones que no se habían concebido antes, ya que no existían las bases técnicas ni la facilidad de construir nuevas alternativas que ahora permite la red (Sassen, 2012). Por lo general, cada una de esas iniciativas pone en cuestión, altera o precariza organizaciones, empresas o posiciones que antes controlaban, muchas veces con pleno respaldo legal e incluso en situación de monopolio, la posibilidad de operar en ese campo. Los ejemplos más claros son los de la llamada economía colaborativa. En ese marco se 
promueve que los propios usuarios sean quienes, de manera agregada, se organicen para conectar directamente con los productores de servicios, o que incluso se autoprovean esos servicios aprovechando las ventajas, bajos costes y operatividad de Internet y de las redes sociales (Benkler, 2015).

Una parte muy significativa de la literatura dedicada al análisis de políticas públicas se centra en examinar los límites de cambio estructural que podían incorporar las iniciativas frente a determinados problemas sociales. Las aportaciones de Lindblom sobre la adecuación de la lógica incremental, para conseguir avanzar ante previsibles bloqueos si se pretendía alterar en exceso los equilibrios existentes, han tenido siempre una gran capacidad descriptiva de los procesos de decisión. Hoy se puede ver cómo, en la propia fase de definición del problema, es cada vez más real la posibilidad de disrupción que implica afrontar una determinada situación desde bases tecnológicas muy distintas, sin tener en cuenta las posiciones de actores e intereses ya existentes. Ello rompe con las posiciones y los repartos de poder trabajosamente construidos durante años. En ese sentido, se podría decir que se impone la necesidad de revisar la literatura relativa a los aspectos relacionados con la definición del problema y la generación de alternativas para diseñar una política pública de respuesta. Precisamente uno de los grandes cambios que introduce Internet es el que se da en los formatos abiertos de generación de alternativas (por ejemplo, la tercerización, la externalización masiva, los procesos entre pares y la programación de código abierto), que rompen con los canales de innovación antes establecidos e institucionalizados.

Los impactos son aún más significativos en la conformación de la agenda social y la agenda de políticas públicas. Si en el caso de la innovación abierta en los procesos productivos se podría decir que apenas comienza el proceso de transformación, los cambios son ya mucho más visibles y constatables en la esfera informativa y comunicativa, que es el marco adecuado de la construcción de la agenda. Temas que hasta hace poco parecían tabúes en los medios de comunicación tradicionales, están hoy presentes en esos mismos medios, simplemente porque nadie puede frenar su aparición ni el debate que se genera en las redes sociales. Ello afecta a intereses de grandes empresas que antes controlaban los medios de comunicación mediante la inversión en publicidad, pero también a instituciones o personas sobre quienes existía cierto pacto de silencio o de discreción. La agenda social y de políticas públicas se construye hoy de manera mucho más abierta, con la presencia de muchos más actores, y con puntos nodales o centros de referencia menos localizables y controlables que antes. Al mismo tiempo, hay nuevos actores que conocen mejor la manera de operar en los espacios digitales y que se valen de lógicas de actuación multicapa (mezclando medios convencionales y redes digitales) para generar impactos significativos en la configuración de esa agenda (Sassen, 2012). 
La esfera de la adopción de decisiones también se ve alterada forzosamente. Las lógicas de acomodación entre actores, perspectivas e intereses que acaban madurando en decisiones que permitían avanzar, aunque fuera de manera incremental, se convierten ahora en procesos más tumultuosos. En ellos no siempre resulta previsible el ritmo que se seguirá en la adopción de decisiones ni el grado de turbulencia que se deberá asumir. Sobre todo, no se puede vislumbrar con certeza el conjunto de actores que deberán incorporarse en el proceso final de toma de decisiones. Al mismo tiempo, empiezan a darse muchas situaciones en que los actores implicados en un problema determinado no siempre esperan a que las instituciones actúen y tomen decisiones. Ellos mismos toman la iniciativa (muchas veces en nombre de los intereses generales) y tratan de resolver o paliar el tema con sus propios recursos y su propia capacidad de agencia.

En ese escenario de desinstitucionalización, o de asunción colectiva de problemas que afectan a una comunidad, las posibilidades que ofrece la red de obtener recursos de todo tipo (económicos, materiales, informativos y comunicativos) resultan especialmente relevantes (por ejemplo, externalización masiva y financiación entre pares). Hay casos concretos relacionados con el tema de la vivienda en España (con la Plataforma de Afectados por la Hipoteca (PAH) y su obra social), así como los temas de los huertos urbanos y las redes de distribución alternativas en muchas partes de Europa. Si se analiza la fase de implementación o puesta en práctica de las decisiones tomadas, también queda claro que no resultará fácil seguir operando con las dinámicas burocráticas de corte tradicional, ni con las perspectivas de mayor eficacia y eficiencia con que se impulsó la idea de la nueva gestión pública (Dunleavy y otros, 2006).

\section{La (fallida) perspectiva de la gobernanza participativa como respuesta}

Desde hace tiempo se detectaron déficits en el funcionamiento de las instituciones representativas, generados en parte por los primeros impactos de la globalización y el cambio tecnológico, como fenómenos paralelos. A ese respecto, tanto desde el punto de vista político como de gestión, se fueron impulsando iniciativas que se vincularon a nuevos formatos de gobernanza.

La gobernanza se presentó como una forma específica de regulación, coordinación y control (Rhodes, 1997) que se fundamentaba en el hecho de incorporar a actores del mercado y de la sociedad civil en el proceso de gobernar. Como indicó Kooiman (1993), se trataba de crear nuevas estructuras y capacidades de gobierno derivadas de la interacción de una multiplicidad de actores influyentes. En ese nuevo paradigma, el 
Estado no era el único actor responsable de la elaboración de las políticas públicas sino que, voluntariamente, compartía esa responsabilidad con otros actores. La gobernanza aparece a finales del siglo XX como respuesta a las dos grandes debilidades de las formas tradicionales de gobierno: su falta de funcionalidad y su falta de legitimidad. La primera, provocada por las rigideces de la burocracia (en un mundo que relaciona cada vez más la calidad con la flexibilidad y la personalización); la segunda, por las crecientes inequidades generadas por el mercado (por la erosión de los equilibrios conseguidos en ciertas partes del mundo entre mercado y redistribución, y los procesos de globalización). En la primera de las perspectivas cabría situar la respuesta que representó la nueva gestión pública. En la segunda, se situaría la respuesta que alimentó la llamada perspectiva de la gobernanza participativa.

Desde la primera línea de cambio mencionada no parecen haberse superado los déficits. Más bien, transcurridos los años, lo que destaca es la falta adecuada de respuesta de la nueva gestión pública en relación con los nuevos retos que plantea la transformación digital. En lugar de generar más capacidad de rendición de cuentas de las administraciones a los ciudadanos, lo que ha ocurrido es una notable difuminación de las responsabilidades debido a la mayor complejidad institucional derivada de los partenariados y la distinción de roles. Por su parte, los posibles avances en materia de eficacia y eficiencia no han compensado esa pérdida de valores democráticos de las instituciones. Lo que ahora se detecta es la necesidad de recuperar la capacidad de dirección pública y de gestión global del archipiélago administrativo generado. En ese sentido, se postula la incorporación de la posibilidades y recursos que la era digital plantea y posibilita (Dunleavy y otros, 2006).

Por otro lado, los cambios provocados más por la falta de legitimidad que por la falta de eficiencia y eficacia han promovido la gobernanza como un nuevo paradigma de regulación que involucre a la ciudadanía en general, y a la sociedad civil organizada en particular, en la elaboración de las políticas públicas. Esa visión participativa de la gobernanza, más orientada a la comunidad que al mercado, partía de las teorías de la democracia participativa y defendía la involucración de la sociedad civil como garantía para evitar las inequidades del mercado. De esa manera se defendía la idea de que la participación de los actores clave y la ciudadanía en general en el proceso de definición del problema, de decisión, de implementación o de evaluación de una política, permitiría un mejor diagnóstico. A la vez, facilitaría la aparición de soluciones creativas e innovadoras. Por otra parte, se hacía hincapié en que la participación de la ciudadanía en los procesos de toma de decisiones públicas podría promover una mayor comprensión del problema y una corresponsabilización respecto de la solución. De ese modo, la política pública finalmente implementada obtendría un mayor grado de legitimidad. 
La gobernanza participativa, en definitiva, se presentaba como una nueva forma de elaborar las políticas públicas. Sin embargo, eso no significa que los resultados de los procesos de gobernanza hayan acabado siendo necesariamente distintos de los procesos tradicionales de gobierno (Stoker, 1998; Peters y Pierre, 1998). En ese contexto, las experiencias de participación ciudadana promovidas por diversas administraciones públicas aumentaron significativamente durante los años previos al actual escenario de crisis económica y financiera. Al mismo tiempo, sin embargo, y en paralelo a ese incremento cuantitativo, se empezó a poner en duda la calidad de esas experiencias y su capacidad real de alcanzar los dos objetivos antes mencionados (Parés, 2009). En ese contexto fue aumentando la necesidad de disponer de instrumentos de evaluación que permitieran definir las fortalezas y debilidades de las experiencias participativas.

El debate sobre cómo evaluar la participación de la ciudadanía en las instituciones públicas emergió en la ciencia política durante la primera década del milenio a nivel internacional. En algunas de las contribuciones a ese debate se centró la atención en la evaluación de los espacios y mecanismos concretos de participación (Anduiza y De Maya, 2005; Fung, 2003; Jorba, Martí y Parés, 2007; Papadopoulus y Warin, 2007), mientras que otros lo abordaron a partir de la llamada gobernanza en red (Bevir, 2010; Mathur y Skelcher, 2007; Sorensen y Torfing, 2005). Así, las nuevas formas de gobernanza participativa impulsadas desde las administraciones públicas, basadas en el análisis de políticas públicas, se acompañaron de nuevos enfoques evaluativos que pusieron el acento en el papel de los actores en general y de la ciudadanía en particular, la articulación más o menos democrática de las redes de gobernanza y el análisis de los mecanismos participativos, tanto en lo relativo a su funcionamiento como a sus impactos en las políticas públicas.

\section{Otra perspectiva: políticas públicas y procesos compartidos de producción e implementación en la era digital}

¿Cómo avanzar en sistemas tecnológicamente mediados de prácticas democráticas que permitan otra concepción de políticas públicas? (Edwards, 2006; Subirats, 2012). ¿Cómo aprovechar las nuevas dinámicas de producción distribuida y compartida en las dinámicas de intervención de los poderes públicos? (Long, 2002). ¿Es posible hablar de "gobierno abierto", de nuevas formas de usar las potencialidades de los datos abiertos? (Ramírez-Alujas, 2011, 2012; Janssen, Charalabidis y Zuiderwijk, 2012). Se necesitan distintos elementos para tratar de responder a esas preguntas aunque sea de forma parcial. De entrada, es necesario 
entender que el cambio digital permite que se abran y amplíen espacios significativos entre la lógica del mercado y la organización propia de los Estados. El espacio de lo común, entendido como la capacidad colectiva de enfrentarse a problemas comunes, con o sin las instituciones, es un espacio que la transformación digital ha permitido ampliar y redimensionar. No es ninguna novedad. Los trabajos de Ostrom (2000) enraizaron desde el punto de vista académico y teórico un conjunto de prácticas ancestrales de algunas comunidades que, a lo largo de los siglos, fueron capaces de mantener de manera sostenible y organizada sus bienes comunes. La labor de Ostrom consistió en recoger esa multiplicidad de prácticas, y luego sistematizar y ordenar sus reglas institucionales, es decir, sus formas de gobierno de lo común. Además, es significativo ver la gran proliferación de nuevas experiencias de innovación productiva que usa formatos de colaboración, reciprocidad y conocimiento compartido, con mecanismos y métricas de generar y medir valor que no son exactamente las mismas que en la economía mercantil (Benkler, 2015; Fuster Morell, 2010; Algan y otros, 2013, Bauwens, 2005). Hay ejemplos de desintermediación y de nuevos formatos de producción, como la economía colaborativa y las dinámicas de la producción entre pares basada en el patrimonio común ${ }^{1}$.

En el campo de las políticas públicas, el debate sobre la incorporación de esas lógicas en la formación, decisión e implementación de las políticas está contribuyendo a que tome forma el concepto de coproducción de políticas y servicios públicos de innovación colaborativa. Esto va más allá de la lógica muchas veces fuertemente tecnocrática y eficientista del gobierno electrónico, que ve en el ciudadano un cliente a quien servir, y no alguien con capacidad para diseñar, decidir e implementar conjuntamente (Scherer, Wimmer y Strykowski, 2015; Koch, Füller y Brunswicker, 2011; Linders, 2012; Sørensen y Torfing, 2012).

El concepto de gobernanza de la era digital (Margetts y Dunleavy, 2013) rompe con la idea - muy presente en la nueva gestión públicade que, en general y para obtener resultados mejores y más eficientes, es mejor generar competencia (se busca así la incorporación de la lógica del mercado en el funcionamiento del sector público). La fuerte tendencia a desagregar estructuras administrativas, generar partenariados 0 buscar fórmulas de cuasimercados respondía a ese criterio. Lo mismo sucedía con la idea-fuerza de generar incentivos de mejora en un entorno en que no estaba presente ese tipo de fórmulas de aumentar la productividad. Lo que se afirma desde la aproximación de la gobernanza de la era digital es que, en las nuevas coordenadas del escenario digital, muchas veces es mejor compartir o colaborar que competir. Además, las métricas de valor pueden circular por otros derroteros que no sean

Véase [en línea] http://igopnet.cc/es/p2pvalue-3/. 
estrictamente monetarios. Existen evidencias que muestran los límites de la aproximación de la nueva gestión pública una vez transcurridos suficientes años como para establecer cierto balance (Margetts y Dunleavy, 2013). Ello conlleva la oportunidad y la necesidad de hacer planteamientos que generen reintegración de servicios, visiones más holísticas del servicio público y un aprovechamiento más sustantivo y menos instrumental de las potencialidades de la digitalización.

Si está comenzando una era en que se busca la mejor combinación posible entre iniciativa y capacidad de innovación social y disponibilidad tecnológica a bajo coste, ¿cómo se puede aprovechar esa combinación para repensar las políticas públicas y la prestación de servicios que esas políticas incorporan? En general, tanto las políticas como los servicios se han pensado de manera jerárquica, desde una lógica competencial segmentada, en la que se considera a los ciudadanos más como objetos de atención que como sujetos de decisión. Toda la dinámica de cambio que caracteriza la era digital se basa en el reconocimiento de que es mucho más eficaz y útil confiar en la capacidad de innovación colectiva que en la concentración de las decisiones sobre diagnósticos, alternativas y soluciones en un grupo de técnicos y expertos, por calificados que sean. La experiencia de cómo Wikipedia se impuso a la Enciclopedia Británica ofrece un pequeño ejemplo a ese respecto (Algan y otros, 2013; Fuster Morell, 2010). A partir de esa convicción ha ido configurándose la idea de coproducción de políticas y servicios públicos (Bason, 2012; Scherer, Wimmer y Strykowski, 2015). Uno de los elementos clave en ese cambio de perspectiva es la consideración de copartícipes de los ciudadanos en una lógica de reciprocidad e interdependencia, no de jerarquía.

Esa concepción de coproducción de servicio estaba ya presente en los trabajos de Ostrom (1996) cuando afirmaba que podían establecerse nuevas estrategias en que la relación que podría existir entre el "productor común" (por ejemplo, los agentes policiales a pie de calle, los trabajadores sociales o los trabajadores de la salud) y sus clientes, que deseaban ser transformados por el servicio en personas más seguras, mejor educadas o más sanas (citado por Brandsen y Pestoff, 2006). Según esa lógica, la posición de los ciudadanos es muy distinta de la de "clientes". Así pues, habría que reflexionar sobre cómo evolucionará la relación entre ciudadanos y sus necesidades, y el grado en que aquellos sean capaces de desplegar mecanismos y vías para encontrar respuestas satisfactorias a estas, o hasta qué punto se seguirán demandando esos servicios de los poderes públicos, aun a costa de mantener una posición de subordinación y sujeción, de pasividad y de no capacidad de intervención. Lógicamente, esa mayor capacidad de innovación, intervención y coproducción de servicios por parte de la ciudadanía y de las organizaciones o movimientos en los que se encuadre debería venir acompañada de una modificación en 
la estructura de poderes y los formatos en que se expresan las relaciones entre poderes públicos e instituciones representativas y dinámicas de innovación social (Moulaert y otros, 2013).

\section{E. Conclusión: algunas reflexiones finales}

$\mathrm{Al}$ principio de esta contribución se planteaba la interrogante de hasta qué punto el cambio tecnológico asociado con Internet (y que no generaba una crisis episódica o coyuntural, sino que tenía dimensión estructural, de cambio de época) no exigía un replanteamiento general del modo de pensar en las formas de gobierno y de cómo entender y analizar las políticas públicas en los sistemas democráticos contemporáneos. El itinerario seguido y las referencias acumuladas permiten concluir que no solo es posible cierto proceso de acomodación, sino que conviene incorporar esa gran transformación de fondo en el esquema de análisis de los gobiernos y las administraciones públicas. Se avanza de un espacio de notable previsibilidad, en que las rutinas procedimentales resultaban útiles, a escenarios de gran innovación, en que estarán cada vez más presentes los procesos basados en conocimiento intensivo y compartido, y en que será cada vez más escasa la posibilidad de seguir itinerarios previamente establecidos. En esos escenarios puede resultar más útil la posibilidad de construir espacios de coproducción de políticas, desde la conformación del diagnóstico hasta la dinámica concreta de implementación. De ese modo se evitan los bloqueos generados por asunciones previas, rutinas preestablecidas o dinámicas de control de espacios propios de las comunidades normativas más cerradas.

La concepción de lo público debería ampliarse para evitar su estricta asimilación a lo institucional. Ha de entenderse la potencia de la lógica de "lo común" como un concepto que permite incorporar ese conocimiento en la dinámica de cambio social y en la posibilidad de gobernar colectivamente la complejidad de cada situación concreta. Ese es el reto: encontrar mejores capacidades descriptivas y analíticas respecto de un escenario en que las políticas públicas perderán buena parte de su carácter jerárquico y lógica técnica, para incorporarse en las lógicas de gobierno abierto, producción distribuida y conocimiento compartido, propias del nuevo escenario digital. 


\section{Bibliografía}

Algan, Y. y otros (2013), "Cooperation in a Peer Production Economy Experimental Evidence from Wikipedia", Workshop on Information Systems and Economics, Milán. Anduiza, E. y S. De Maya (2005), La Qualitat en la Participació: una proposta d'inicadors, Barcelona, Fundació Jaume Bofill.

Bason, C. (2012), "Designing co-production: discovering new business models for public services", Leading Through Design, 311.

Bauman, Z. (2012), “Times of interregnum”, Ethics E Global Politics, vol. 5, No 1.

Bauwens, M. (2005), “The political economy of peer production”, CTheory [en línea] http: / / www.ctheory.net/articles.aspx?id=499.

Benkler, Y. (2015), La riqueza de las redes. Como la producción social transforma los mercados y la libertad, Barcelona, Icaria.

Bennett, W. L. y S. Iyengar (2008), "A new era of minimal effects? The changing foundations of political communication", Journal of Communication, vol. 58, $\mathrm{N}^{\mathrm{o}} 4$.

Bennett, W. L. y A. Segerberg (2012), "The logic of connective action: Digital media and the personalization of contentious politics", Information, Communication and Society, vol. 15, No 5 .

Bevir, M. (2010), Democratic Governance, Princeton, Princeton University Press.

Castells, M. (2009), Comunicación y poder, Madrid, Alianza Editorial.

Brandsen, T. y V. Pestoff (2006), "Co-production, the third sector and the delivery of public services: An introduction", Public Management Review, vol. 8, No 4 .

Chadwick, A. y Ph. Howard (eds.) (2009), The Handbook of Internet Politics, Londres, Routledge.

Chircu, A. M. y R. J. Kauffman (1999), "Strategies for Internet middlemen in the intermediation/disintermediation/reintermediation cycle", Electronic Markets, vol. $9, \mathrm{~N}^{\mathrm{o}} 1-2$.

Deibert, R. J. (2000), “International plug'n play? Citizen activism, the Internet, and global public policy", International Studies Perspectives, vol. 1, No 3 .

Dente, B. y J. Subirats (2014), Decisiones públicas. Análisis y estudio de los procesos de decisión en políticas públicas, Barcelona, Ariel.

Di Maggio, P. y otros (2001), "Social implications of the Internet", Annual Review of Sociology, $\mathrm{N}^{\mathrm{o}} 27$.

Dunleavy, P. y otros (2006), "New public management is dead -long live digitalera governance", Journal of Public Administration Research and Theory, vol. 16, $\mathrm{N}^{\mathrm{o}}$ 3.Edwards, A. (2006), "ICT Strategies of Democratic Intermediaries", Information Polity, $\mathrm{N}^{\mathrm{o}} 11$.

Font, J. (2001), Ciudadanos y decisiones públicas, Barcelona, Ariel.

Fung, A. (2003), "Survey Article: Recipes for Public Spheres: Eight institutional design choices and their consequences", The Journal of Political Philosophy, vol. $11, \mathrm{~N}^{\mathrm{o}} 3$.

Fuster Morell, M. (2010), “Governance of online creation communities: Provision of infrastructure for the building of digital commons", tesis de doctorada.

Gellman, R. (1996), "Disintermediation and the Internet", Government Information Quarterly, vol. 13, No 1 .

Hacker, K.L. y J.Van Dijk (2000), "What is digital democracy?", Digital Democracy. Issues of Theory and Practice, K.L. Hacker y J. van Dijk (eds.), Londres, Sage Publications.

Jellinek, G. (1978), Fragmentos de Estado, Madrid, Civitas. 
Janssen, M., Y. Charalabidis y A. Zuiderwijk (2012), “Benefits, adoption barriers and myths of open data and open government", Information Systems Management, vol. 29, $\mathrm{N}^{\mathrm{o}} 4$.

Jorba, L., J. Martí y M. Parés (2007), La qualitat en la participació: orientacions per a l'avaluació participada, Barcelona, Fundació Bofill.

Koch, G., J. Füller y S. Brunswicker (2011), "Online crowdsourcing in the public sector: how to design open government platforms", Online Communities and Social Computing, Heidelberg, Springer.

Kooiman, J. (1993), Modern Governance: Government Society-Interactions, Londres, Sage.

Linders, D. (2012), "From e-government to we-government: Defining a typology for citizen coproduction in the age of social media", Government Information Quarterly, vol. 29, $\mathrm{N}^{\mathrm{o}} 4$.

Long, M. (2002), "Beyond traditional boundaries: Government in the information age", Australian Journal of Public Administration, vol. 61, No 1 .

Margetts, H. Z. (2009), "The Internet and public policy", Policy and Internet, vol. 1, Nº 1.

Margetts, H.Z. y P. Dunleavy (2013), "The second wave of digital-era governance: a quasi-paradigm for government on the Web", Philosophical Transactions of Royal Society, vol. 371.

Mathur, N.y C.Skelcher(2007), “Evaluating democratic performance: methodologies for assessing the relationship between network governance and citizens", Public Administration Review, vol. 67, $\mathrm{N}^{\mathrm{O}} 2$.

Mayntz, R. y F.W. Scharpf (1975), Policy-making in the German federal bureaucracy, Elsevier.

Morozov, E., (2014), To Save Everything, Click Here. The folly of technological solutionism, Londres, Public Affairs Books. (2012), The Net Delusion: The Dark Side of Internet Freedom, Londres, Public Affairs Books.

Moulaert, F. y otros (eds.) (2013), The International Handbook on Social Innovation: collective action, social learning and transdisciplinary research, Edward Elgar.

Ostrom, E. (2000), El gobierno de los bienes comunes: la evolución de las instituciones de acción colectiva, Ciudad de México, Fondo de Cultura Económica de México. (1996), "Crossing the great divide: coproduction, synergy, and development", World Development, vol. 24, Nº 6 .

Oszlak, O. y otros (1976), Estado y políticas estatales en América Latina; hacia una estrategia de investigación. Conferencia sobre Políticas Públicas y sus Impactos en América Latina (INVES-ET D10 086e), Buenos Aires, Centro de Estudios de Estado y Sociedad.

Papadopoulos, Y. y P. Warin (2007), “Are innovative, participatory and deliberative procedures in policy making democratic and effective?", European Journal of Political Research, vol. 46.

Parés, M. (2009), Participación y calidad democrática. Evaluando las nuevas formas de democracia participativa, Barcelona, Ariel.

Parsons, D. W. (1995), Public Policy: an introduction to the theory and practice of policy analysis, Edward Elgar.

Peters, B. G. (2012), American Public Policy: Promise and performance, Cq Press.

Peters, B. G. y J. Pierre (1998), “Governance without government? Rethinking public administration", Journal of Public Administration Research and Theory, vol. 8, No 2.

Pitkin, H. (2014), El concepto de representación, Madrid, CEPC. 
Poster, M. (2007), "Internet Piracy as Radical Democracy", Radical Democracy and the Internet. Interrogating Theory and Practice, L. Dahlberg y E. Siapera (eds.), Nueva York, Palgrave-Macmillan.

Radaelli, C. M. (2003), "The Europeanization of public policy", The Politics of Europeanization, K. Featherstone y C. M. Radaelli (eds.), Oxford.

Ramírez-Alujas, A. V. (2011), "Gobierno abierto y modernización de la gestión pública. Tendencias actuales y el (inevitable) camino que viene-reflexiones seminales", Revista Enfoques: Ciencia Política y Administración Pública, vol. 9, No 15. (2012), "Gobierno abierto es la respuesta: ¿Cuál era la pregunta?", Más Poder Local, $\mathrm{N}^{\mathrm{o}} 12$.

Reinicke, W. H., y D. Copeland (1998), "Global Public Policy: Governing without Government?", International Journal, vol. 53, No 3 .

Rhodes R.A.W (1997), Understanding governance: policy networks, governance, reflexivity, and accountability, Open University Press.

Sassen, S. (2012), "Interactions of the technical and the social. Digital formation of the powerful and the powerless", Information, Communication and Society, vol.15, No 4 .

Scherer, S., M. A. Wimmer y S. Strykowski (2015), "Social government: a concept supporting communities in co-creation and co-production of public services", Proceedings of the 16th Annual International Conference on Digital Government Research, ACM.

Schmidt, V. A. y C.M. Radaelli (2004), "Policy change and discourse in Europe: Conceptual and methodological issues", West European Politics, vol. 27, N 2 .

Sørensen, E. y J. Torfing (2011), "Enhancing collaborative innovation in the public sector", Administration and Society, vol. 43, $\mathrm{N}^{\mathrm{o}} 8$.

(2005), "The democratic anchorage of governance networks", Scandinavian Political Studies, vol. 28, $\mathrm{N}^{\circ} 3$.

Stoker, G. (1998), "Governance as a theory: five positions", International social Science Journal, vol. 50, $\mathrm{N}^{\mathrm{o}} 155$.

Subirats, J. (2012), “Nuevos tiempos, ¿nuevas políticas públicas? Explorando caminos de respuesta", Reforma y Democracia, $\mathrm{N}^{\mathrm{o}}$ 54, Caracas [en línea] http:/ / www.clad.org/portal/publicaciones-del-clad/revista-clad-reformademocracia/articulos/054-octubre-2012/Subirats.pdf.

(2011), Otra sociedad ¿otra política? De «no nos representan» a la democracia de lo común, Barcelona, Icaria Asaco.

(2002), "The dilemmas of an inevitable relationship: democratic innovation and the information and communication technology", Governing Telecommunications and the New Information Society in Europe, J. Jordana (ed.), Edward Elgar.

(1989), Análisis de políticas públicas y eficacia de la administración, Instituto Nacional de Administración Pública (INAP).

Subirats, J. y otros (2008), Análisis y gestión de políticas públicas, Ariel.

The Economist (2012), "The Third Industrial Revolution", 21 de abril [en línea] http://www.economist.com/node/21552901. 